\title{
LAS ESCUELAS UNIDOCENTES RURALES COMO ORGANIZACIONES DE CAMBIO
}

El artículo revela que las escuelas unidocentes surgen, en la década del setenta, como producto de una circunstancia histórica: la necesidad de reducir el personal docente en concordancia con la cantidad de alumnos. Esta disposición se llevó a cabo sin asegurarse la calidad del servicio escolar (infraestructura o material didáctico, por mencionar algunos aspectos). A raíz de estas circunstancias, la autora reflexiona acerca del compromiso que las universidades deberian tener con las escuelas unidocentes, asi como el perfil que el maestro que asume ese puesto. Finaliza con un listado de desafios que estos centros educativos pueden tener presentes para ejercer una función transformadora en la comunidad rural.
The article reveals that multi-grade instruction schools emerged during the 1970s as a product of historical circumstances: the need to reduce the number of teachers according to the number of students. This decision was applied without providing for the quality of the school services (such as infrastructure or instructional material). Based on these experiences, the author analyzes the commitment that the universities must make with schools with only one teacher, as well as the profile of the teacher assuming the position. She concludes with a list of challenges that these schools should keep in mind so that they can contribute to the transformation of rural communities.

* Obtuvo su maestría en Administración Educativa en la Universidad de La Salle. Actualmente es asesora de la Subgerencia Administrativa del Instituto Nacional de Aprendizaje (INA), participa en el Proyecto de Administración Efectiva de la Educación en el Contexto Rural y labora como académica de la División de Educación Rural del CIDE.jperez@racsa.co.cr. 
En estos tiempos de incertidumbre, donde la urgencia por una reestructuración y reconversión profunda del sistema educativo es descuidada por las autoridades, y archivada por la mayoría de las instituciones de educación superior encargadas de la formación de docentes, los centros educativos en general y particularmente aquellos que se desarrollan en el ámbito rural se ven obligados a aprender a responder a las demandas de un entorno incierto, turbulento, inestable, sin esperar ni confiar en reformas estructurales.

Se debe recordar que, por su parte, las escuelas unidocentes son producto de una circunstancia histórica y de una definición particular. Para una administración que a partir de la década de los setenta se propuso la expansión del nivel primario, el problema consistía en asegurar la existencia del servicio escolar, sin que por entonces su calidad fuera objeto de preocupación.

Como en otros países de la región, esta fue la manera en que Costa Rica respondió a las particularidades de la demanda rural, sin atender a la naturaleza de la acción pedagógica que se requería. La estructura conocida y disponible de escuela, nacida para medios urbanos, se consideró pertinente para ser aplicada al medio rural con escasa y heterogénea población infantil. La escuela unidocente, más que respuesta y estrategia pedagógica, en este caso fue el resultado de una operación administrativa que, para brindar el servicio, encontró la solución de reducir el personal en concordancia con la cantidad de alumnos. La práctica pedagógica, los contenidos curriculares y las obligaciones administrativas quedaron sometidos a las regulaciones propias de otra realidad escolar, la urbana. con un maestro por grado. $Y$ debo añadir que estas obligaciones administrativas en manos de las pequeñas escuelas son una carga pesada que ahoga a directores y docentes.

Es así como deseo referirme, ya no a las estrategias burocráticas, verticales o racionales de cambio, sino a aquellas referidas a dinámicas autónomas de progreso, que devuelven de una u otra manera el protagonismo a los maestros y a los directores de las escuelas y por ello pudieran tener un mayor grado de permanencia.

Hemos entendido, en el contexto del trabajo de capacitación con directores de centros educativos rurales, que no se puede esperar la reforma del sistema educativo para hacerle frente a las necesidades que el cambiante entorno presenta a los centros educativos: no será con decisiones ministeriales que la escuela rural puede convertirse en una organización que aglutine la sinergia comunitaria, para el beneficio de sus habitantes.

Es por esta razón que se propone la transformación de los centros educativos desencadenando procesos de autodesarrollo, que tengan un grado de permanencia. La capacitación que se brinda a directores y docentes de zona rural -en 
el marco del Proyecto de Administración Efectiva de la Educación para Escuelas Rurales- tiene una propuesta que pretende promover la capacidad de desarrollo de la organización y de las escuelas como centros de organización con sinergia dentro de la comunidad en que se desenvuelve.

Incorporar una nueva visión de la gestión a las escuelas rurales supone, al mismo tiempo, ayudar a generar la propia noción de gestión en el sistema educativo y tratar de adecuar ésta a las formas tradicionales de administración, con el fin de seguir interactuando con el sistema de manera eficaz.

Vale la pena recordar que, de todas las regulaciones vigentes sobre ellas, incluidas las pedagógicas, las que se materializan como controles explícitos son aquellas que responden a la necesidad administrativa. Las directrices de carácter pedagógico quedan un poco en el ámbito de la "asesoría", de la "propuesta", y en poco o ningún caso llevan a la interiorización de formas específicas de ver la administración del pensum curricular.

\section{Introducción}

Atendiendo a sus rasgos más formales, generales y, sobre todo, comunes, el control ejercido sobre las escuelas rurales las ha conducido a forzar su naturaleza múltiple y diversa. Con el transcurso del tiempo y el crecimiento de la burocratización, ajustarse a los parámetros de homogeneidad ha implicado para ellas debilitar el sentido sustantivo de su trabajo, desalentar la iniciativa pedagógica y, a menudo, refugiarse en la simulación. La propuesta planteada en esta ponencia cuestiona, de hecho, la preponderancia de ese enfoque que, a la vez, es indisociable de la crisis de calidad que a todas luces se conoce.

Cuando la calidad del servicio educativo que se ofrece adquiere mayor relevancia política que su expansión, lo que se está enunciando e implicando es un desplazamiento de eje en la visión del sistema educativo. Si el logro de la calidad implica atender el manejo de las escuelas, se deben construir criterios para dar contenido a las necesidades de un nuevo objeto de la preocupación política: regular y administrar instituciones y condiciones de práctica pedagógica. Esto por sí mismo, abre un horizonte distinto al de regular y administrar la prestación del servicio educativo (aunque la costumbre haya podido confundif lo con regulación, también de la práctica pedagógica), para lo cual el enfoque administrativo, sin duda pudo haber sido adecuado.

Junto a la construcción de criterios orientados hacia la calidad, el desafío paralelo, y tal vez mayor, pareciera situarse en la redefinición del papel y de los criterios administrativos, sin olvidar que éstos llevan la ventaja de ser los únicos vigentes $y$, a la vez, de dar cuenta de un sector de la realidad del sistema que no 
ha dejado de tener vigencia, a pesar de la importancia que se le quiere dar a la calidad de la educacion.

Los administradores educativos, con puestos en diferentes niveles del sistema, suelen hablar de panoramas confusos. A veces parecieran pensar que las actuales estructuras, con sus antiguas pautas, pueden ser "mejoradas" agregándoles, aquí y allá, elementos de la nueva perspectiva, dejando de lado que su origen en "otro objeto" las hace muy débilmente articulables. Otras veces, la noción de gestión se interpreta como el trasplante de saberes propios del orden de la economía para sólo potenciar la perspectiva administrativa, o bien, para pensar en el sistema y sus escuelas como réplicas de la empresa.

Si la gestion puede entenderse como el conjunto de estrategias para acercar las formas de operación institucional del servicio educativo a la finalidad específica de la escuela - ofrecer práctica pedagógica y provocar aprendizajes-, se estaría revitalizando el papel de la escuela, del mismo modo que se estaría introduciendo la aceptación del dinamismo y la diversidad que caracterizan a los establecimientos escolares y las comunidades en las cuales están insertas.

En tanto estas dimensiones carecen de interés para las regulaciones vigentes en las escuelas primarias en general, y en particular para las rurales unidocentes, esta reflexion intenta perfilar algunos núcleos de problemas significativos que deben tomarse en cuenta si se desean establecer nuevas orientaciones de las políticas.

Entre las dimensiones identificadas me permitiré señalar tres, por considerarlas significativas. Se tratará, en primer término, de la especificidad que tienen las instituciones que laboran bajo el esquema de trabajo unidocente: luego se revisarán las características del acceso a la docencia rural para, finalmente, considerar las políticas de atención o refuerzo a la práctica pedagógica.

\section{La especificidad institucional de la escuela unidocente}

Los programas escolares para las escuelas unidocentes están concebidos y estructurados para el trabajo con diversos grados de manera simultánea. Si se conversa con los maestros, ellos destacan este hecho, que, siendo obvio para el interés pedagógico, nunca lo fue para la organización del sistema escolar: que el núcleo de la actividad institucional es la práctica pedagógica y, simultáneamente, que el trabajo con pocos niños con distintos niveles de avance requiere una resolución organizativa diferenciada respecto del modelo de un maestro por grado, con grupos relativamente homogéneos.

Por su parte, las escuelas unidocentes son producto de otra circunstancia histórica: para una administración que a partir de la década de los setenta se 
Los programas escolares para las escuelas unidocentes están concebidos y estructurados para el trabajo con diversos grados de manera simultánea. Si se conversa con los maestros, ellos destacan este hecho, que, siendo obvio para el interés pedagógico, nunca lo fue para la organización del sistema escolar: que el nucleo de la actividad institucional es la práctica pedagógica y, simultáneamente, que el trabajo con pocos niños con distintos niveles de avance requiere una resolu. ción organizativa diferenciada respecto del modelo de un maestro por grado, con grupos relativamente homogéneos. propuso la expansión del nivel primario, el problema consistia en asegurar la existencia del servicio escolar, sin que por entonces su calidad fuera objeto de preocupación.

Como en otros países de la región, esta fue la manera de responder a las particularidades de la demanda rural, sin atender a la naturaleza de la acción pedagógica que ella requería. La estructura conocida y disponible de escuela, nacida para medios urbanos, se consideró pertinente para aplicarla al medio rural con escasa población infantil. La unidocencia, más que respuesta y estrategia pedagógica, en este caso fue el resultado de una operación administrativa que, para brindar el servicio, encontró la solución de reducir el personal en concordancia con la cantidad de

alumnos. La práctica pedagógica, los contenidos curriculares y las obligaciones administrativas quedaron sometidos a las regulaciones propias de otra realidad escolar, la urbana, con un maestro por grado.

Lo mismo que sus colegas de las escuelas completas, los maestros de las escuelas unidocentes trabajan con el plan de estudios y los programas para cada uno de los seis grados de la escuela primaria y con el material didáctico utilizado para cada grado. Con esos materiales ellos necesitan resolver, de forma individual, una serie de operaciones técnicas que el modelo pedagógico imperante les exige. Al menos idealmente se espera que cada maestro decida, en primer lugar, sobre la necesaria selección de contenidos -puesto que es imposible pensar en cubrir el currículum completo para cada grado-; en segundo lugar, sobre la articulación temática de esos contenidos, necesaria para facilitar; en tercer término, sobre la articulación didáctica, que permitiría coordinar las actividades de todos los grados.

Esta "natural" exigencia profesional, derivada del desconocimiento de las exigencias pedagógicas de la unidocencia, se relaciona con otro importante 
criterio implícito en la administración escolar, referido a su confianza en la preparación profesional de los docentes, la cual les permitiria resolver estas operaciones.

El criterio administrativo como eje de las políticas pudo obviar no sólo la reconocida pobreza de esa formación, sino también el hecho de que ésta no incluye la preparación para el unidocente, en los planes de estudio de las universidades. Un esfuerzo particular, en este sentido, ha desarrollado la División de Educación Rural de la Universidad Nacional, tratando de teorizar en relación con el tema de la ruralidad y de incorporar en los planes de estudios de los docentes cursos relativos a la administración de la educación rural, indefectiblemente adscritos al tema de la unidocencia.

En la observación del trabajo en el aula no es difícil advertir las distintas concepciones de organización de la práctica pedagógica subyacentes en el currículo. Formados en la representación de su trabajo para el manejo de un solo grado, los maestros en la situación de unidocentes parecen tendencialmente inclinados a percibir los grados por separado, mientras libran una ardua batalla, pocas veces exitosa, por coordinar las actividades del conjunto. Por su parte, los docentes con algunos años de experiencia en la unidocencia, aunque no dispongan de una propuesta curricular que los sustente, tienden con naturalidad a organizar a los niños por niveles que agrupan a dos grados, mientras libran su batalla por la diferenciación interna de contenidos y actividades dentro de esos dos grados, logrando en pocas ocasiones coordinar el trabajo conjunto de todos los niveles.

Una pregunta que podría surgir en este punto lleva a pensar qué pesa más en el aprendizaje de los niños: la formación propia de los maestros o la disponibilidad de un currículo y de una preparación focalizados, aunque fuese impartida por maestros sin experiencia y con escasa formación. Dentro de la complejidad que presenta la unidocencia en el medio rural, no parece pertinente aislar la formación de otros elementos presentes en el contexto de la inserción docente en la vida de sus respectivos centros. Entre esos elementos es preciso considerar las condiciones que se analizan en los párrafos siguientes.

Habría que agregar aquí las deficiencias en infraestructura -edificaciones en mal estado, escasez de mobiliario y éste en condiciones deplorables-, la carencia del mínimo recurso en material didáctico -sin hablar de libros, revistas o juegos didácticos- que hacen de las condiciones un medio muy difícil para el trabajo de la gestión de la práctica pedagógica.

\subsection{Los docentes y su inserción}

El interés y la expectativa con los que se asume el trabajo en las zonas rurales y en su forma unidocente, diferencian a los maestros. Su atención se 
justifica no sólo para identificar elementos que gravitan en el desempeño, sino fundamentalmente para estimar las políticas que se ponen en juego para facilitar su integración a las estructuras institucionales.

La práctica docente en el contexto de la escuela primaria, nos muestra que, en la docencia -como en el resto de las ocupaciones-, la relación del maestro con su profesión expresa la confluencia de diversos elementos. Entre ellos, pueden señalarse el que sigue: los docentes tienen un vínculo laboral con la escuela, basado en la necesidad económica del trabajo. Esta dimensión no debería ser omitida por los análisis pedagógicos (cuyo fundamento en el supuesto de la vocación instala un silencio sobre el tema que parece acercarlo al territorio de lo ilegítimo). Esto hace que no sea casualidad la escasa atención que los maestros le prestan a las propuestas para pensar y actuar de nuevos modos.

\subsection{Formación universitaria}

Asimismo, los maestros presentan una relación básica con la docencia que, coincidente o no con la necesidad de empleo, toca sus motivaciones individuales más profundas, y que en la escuela se expresa en las formas de asumirla y ejercerla. Este tipo de motivaciones individuales se reconoce entre los docentes dentro de una gama que va desde lo que se identifica como "vocación" hasta lo que es caracterizado como notable descompromiso con la función. Sin embargo, esta relación básica se articula de diversos modos con la formación universitaria recibida, que también presenta gamas. Sus extremos localizan, por un lado, aquella sólida preparación que provee de recursos teórico-prácticos para resolver la tarea pedagógica y, por otro, la más débil formación que provee -en unos cuantos trimestres o bimestres de elevado costo económico- un vacío de competencias profesionales.

Sobre estas distinciones, el contacto permanente con maestros de zona rural permite advertir que la trayectoria escolar más amplia muestra más claramente sus ventajas en aquellos casos que manifiestan un definido interés personal por su profesión. Dentro de la carencia tradicional de instancias que en la escuela propicien la reflexión sobre la práctica, es esa relación individual con su trabajo la que impulsa a algunos docentes a pensarla y problematizarla. Mientras tanto, entre quienes combinan un escaso interés por la docencia con una gran necesidad de empleo, su formación no parece influir en forma significativa para resolver su desempeño. Este amplio margen para la resolución de la tarea pedagógica se inscribe dentro de las tareas administrativas que organizan la vida institucional de cada escuela. Como en todo empleo, la necesidad de conservarlo condiciona el ajuste a sus exigencias. Y las reglas de la institución-Ministerio 
de Educación Pública (MEP) - las que pautan las articulaciones posibles con los motivos individuales para estar en el trabajo.

Si bien la organización institucional y su gestión no pueden incidir en lo que hemos llamado las motivaciones personales más profundas, influyen en cambio en la constitución de las prácticas profesionales y laborales. En este sentido, hay claridad con respecto al poder de las reglas escritas y no escritas vigentes (no necesariamente todas las reglamentadas) que ordenan y permean la vida de la escuela, y de las cuales se apropian los maestros para integrarse y permanecer en su trabajo. Ellas configuran sentidos y representaciones compartidos sobre lo posible y admitido y sobre sus márgenes de legitimidad.

\subsection{La ubicación rural de los maestros}

Parece advertirse que, cada vez más en nuestro país y de manera desafortunada, el origen social de los maestros tiende a concentrarse progresivamente entre las capas medias bajas y bajas de la escala socioeconómica. En las regiones caracterizadas por la ruralidad, crece el ingreso al magisterio de jóvenes de procedencia campesina o de familias del sector informal de la economía. Sin embargo, los maestros costarricenses -en su mayoría- han logrado obtener un título que les habilita para hacer una carrera. Su ingreso al magisterio es el comienzo de una carrera que pareciera prometer destinos mejores.

Para la asignación de los destinos geográficos de los maestros, las zonas rurales pueden valorarse según los grados de aislamiento, acceso a los servicios y posibilidades de comunicación. Lo anterior lo sabe el maestro, que opta por csas regiones de manera voluntaria. Sabe también que a mayor antiguiedad y cursos de capacitación, las posibilidades de desplazarse de una zona lejana e insalubre a las cabeceras de cantón y a la Gran Área Metropolitana, son mayores. De acuerdo con esa lógica, las escuelas unidocentes y rurales, en general, constituyen los destinos obligados para los maestros ingresantes, con poca antiguiedad o "castigados". Esta valoración de las escuelas rurales tradicionalmente vigente, las configura y legitima como instancias "de tránsito". No existe en Costa Rica -como en otros países-el establecimiento de un período mínimo, de hecho no parece posible estimar cuál es la duración de ese período.

El vínculo laboral establecido, sin referencia al núcleo pedagógico de la tarea, plantea un problema importante para el aprendizaje de los niños, que es la aguda movilidad del personal dentro de un año lectivo. Para la continuidad del trabajo escolar, puede decirse que las reglas del Ministerio empleador ponen límites muy débiles al interés individual de los maestros por conseguir destinos más convenientes. Éstos logran sus traslados al margen de la cantidad de cambios 
de docentes que toque a un mismo grupo de niños en un solo año escolar. Es decir. al margen del impacto que las políticas de personal provoquen en el servicio.

La gran mayoría de los maestros no vive en los lugares donde prestan sus servicios y se trasladan a diario o semanalmente. lo cual afecta el horario de trabajo en aula y alimenta las posibilidades de inasistencia.

El conjunto de rasgos hasta aquí señalados alude directamente a criterios del funcionamiento institucional, diferenciados por la mayor o menor atención a las necesidades del trabajo educativo. Esto sin mencionar el consabido compadrazgo político que define los nombramientos en las zonas rurales, de personal sin título pero adscrito al partido político en el poder.

\section{La capacitación a los docentes}

Los apoyos que reciben los maestros bajo la forma de capacitación para fortalecer los procesos de práctica pedagógica y de aprendizaje pueden caracterizarse alrededor de los rasgos de sistematicidad en la capacitación y el seguimiento, y de la concordancia entre esos apoyos y los controles establecidos. Lamentablemente, cada vez más se han perdido los espacios de capacitación que alcanzan a los maestros unidocentes. Para los maestros, como para cualquier trabajador, la presencia de controles constituye un llamado de atención sobre la importancia que ciertos núcleos de su actividad adquieren para el interés de la institución. En el caso de las escuelas, la confluencia de las políticas de apoyo y de control es un articulador clave para estimar el lugar central o subordinado de la práctica pedagógica en las políticas establecidas por el Ministerio.

El asesor supervisor es para las escuelas la referencia directa de autoridad técnica, administrativa y laboral, que canaliza las políticas de apoyo y de control. Los supervisores muestran una vasta experiencia en el servicio: han sido maestros y, a menudo, se han formado como administradores educativos. Son nombrados por el Ministerio en atención a su experiencia y carrera educativa y, en algunos casos -doloroso es decirlo- su filiación política.

Las normas establecidas reservan para la función del asesor supervisor responsabilidades de control administrativo y de apoyo a la práctica pedagógica. En la práctica, el contenido de trabajo que lo caracteriza reconoce dos temas centrales: la administración del servicio y el control del personal. Junto a ellos existe un núcleo periférico que, en términos amplios, podría referirse a la práctica pedagógica. Está constituido mayoritariamente por la difusión de las disposiciones técnico-pedagógicas (y la posterior solicitud de información sobre su recepción o instrumentación) y, secundariamente, por la participación en acciones de capacitación docente. 
El apoyo a la práctica pedagógica, como se mencionó, ocupa un lugar periférico en las funciones del cargo del asesor supervisor. Es así que solamente los unidocentes que deseen en su tiempo privado acudir a universidades públicas o privadas tienen acceso a programas educativos o a cursos que enseñan de manera más o menos articulada nuevas teorías referidas a la práctica pedagógica o al aprendizaje, saberes que en ningún caso se consolidan como obligatorios de su quehacer.

La importancia que tenía hace años atrás la reunión mensual sobre temas de práctica pedagógica con los macstros unidocentes de una misma zona ha sido minimizada y modificada por el nuevo lugar que se adjudica al trabajo sobre los contenidos curriculares, y el cumplimiento del compromiso de los doscientos días efectivos de clase. Cuando se abren espacios en el marco del Proyecto de Administración, esas reuniones parecen tener significación para los maestros. Se ofrece con cllos un espacio para expresar y compartir las inquietudes específicas referidas al unidocente. El logro de estos espacios, se aproxima, más que las visitas del asesor supervisor, a una instancia de refuerzo profesional en vías de construcción.

En el "Proyecto de Administración Efectiva de la Educación en las Escuelas Rurales", el trabajo con algunos asesores supervisores deja entrever el interés personal de éstos por el quehacer propiamente pedagógico. Algunos tratan el tema con regularidad y otros lo suspenden a menudo para atender otras demandas. Algunos reúnen por separado a las escuelas completas y a las unidocentes para propiciar mejores condiciones de análisis y discusión; otros no proponen ninguna forma de diferenciación. Los asuntos administrativos ocupan sistemáticamente algún tiempo en estas sesiones. A veces la presión de la urgencia burocrática parece justificarlo; otras, ofrece buenas coartadas para no abof dar los temas técnicos.

Finalmente no hay que ignorar que los cursos otorgan puntos para la carrera docente y ello asegura simultáneamente su demanda y su permanencia como oferta de actualización. Estas dos circunstancias permitirian ajustar sus contenidos a la nueva perspectiva de gestión institucional.

Poco a poco, los cursos ofrecidos por las universidades públicas y privadas en los que participan docentes de las zonas rurales favorecen la implantación con profundidad y alcances heterogéneos- de una dimensión pedagógica de! trabajo que la función del maestro no tenía incorporada con esta intensidad, aunque parcial y formalmente la incluia. Es previsible que los cambios de concepción y hábitos de trabajo en las personas, así como las prácticas institucionales -cuyo significado es compartido-, acusen durante cierto tiempo una difusión irregular y heterogénea como la observada. Sin embargo, hay elementos 
para preguntarse hasta dónde los cambios percibidos en algunos casos se asocian más al interés individual, docente, de algunos o al efecto de novedad de las propuestas, que al natural ritmo irregular de los cambios. La tendencia deseable de aumentar las responsabilidades pedagógicas de la función del unidocente, disminuyendo la tarea en la administración, plantea la necesidad de reformular los contenidos del cargo, así como los controles que pesan sobre él. Y. de paso, plantea otro buen ejemplo entre el choque de lógicas que responden a la coexistencia de ejes articuladores diversos para gestionar el nivel primario, según se mencionaba al comienzo.

Para terminar, se puede afirmar que la relación de los maestros unidocentes con la estructura institucional de su trabajo, en lo que hace al contenido profesional de la docencia, presenta características notablementedistintas a las de los docentes regulares. A diferencia de los criterios de la gestión señalados para éstos, puede concluirse que, en primer término, en las escuelas unidocentes, el cumplimiento con el trabajo y el desempeño docentes constituyen una referencia mediata a efectos de apoyo y control. En segundo lugar, que el apoyo y el control corren por canales separados. En tercer término, que los referentes de autoridad, claramente explicitados en la normativa, son mediados por prácticas que los tornan ambiguos.

\section{Desafios para el cambio}

Visto el panorama anterior, estos son algunos de los desafios que encuentran las escuelas unidocentes:

- Sería muy importante que la Asesoría para las Escuelas Unidocentes del MEP, iniciara un trabajo de transformación del perfil requerido para los unidocentes. De esta manera, la incidencia de su asesoria trascendería a todas las escuelas unidocentes. Organizar -con el ala administrativa de! Ministerio- las condiciones de trabajo para que sean factibles.

- Primar la tarea efectivamente pedagógica sobre las tareas administrativas impuestas por circunstancias que ya fueron señaladas.

- Tornar a la comunidad como referente para la fijación de metas de la escuela: para lo cual el compromiso con ésta debe ser mucho más fuerte que el vínculo laboral de un año "mientras se produce el traslado". Para lograr este propósito, el MEP puede y debe tomar medidas al respecto.

- Favorecer el advenimiento de los espacios, con el fin de compartir experiencias entre los unidocentes como formas de apoyo a la labor en las comunidades alejadas. $Y$ fortalecer la aparición de perfiles educativos 
propios para cada escuela en función de las necesidades que las comunidades tengan, y no sobre el etéreo concepto de "Proyecto Educativo". Reforzar, tal como se lo ha propuesto la Reforma Educativa Chilena:

- Fortalecimiento de la formación de los profesores en el campo de la educación rural, estableciendo vínculos entre los entes interesados en el tema de la ruralidad y haciendo de las instituciones educativas participantes activos y proactivos en los foros de discusión que se gestan a la luz del desarrollo de las comunidades.

- Desarrollo de un nuevo rol docente como constructor de currículum. Dejando que sea el propio desarrollo de la currícula la que vaya dando las pautas a estos docentes de la organización de su labor, evitando el enfoque administrativista, y favoreciendo los espacios de reflexión y de construcción.

- Desarrollo de una nueva organización escolar abierta a la comunidad. Donde las perspectivas de una apropiada participación comunal vayan más allá de la reparación de las instalaciones... que se conviertan en poseedores de sus propias escuelas para construir un "Proyecto Educativo" legítimo.

- Construcción del proyecto educativo y curricular como respuesta a las necesidades específicas de las comunidades en la que está inserto el centro educativo, como agente de cambio de esa misma comunidad con la flexibilidad necesaria para que pueda continuar independientemente de los cambios de docentes que se susciten.

Para terminar quiero agradecer a la Universidad Nacional, a la División de Educación Rural y al gobierno holandés, así como al personal de Hogeschool Leiden, por el decidido apoyo que ha dado siempre al desarrollo de la ruralidad desde la perspectiva educativa. Espero haber contribuido al menos con algunos referentes, a la discusión de estos temas.

\section{Referencias}

Aldana, L. A. "La Calidad de las escuelas rurales" Artículo publicado en La Prensa en el WEB, 6 de agosto 2000. Honduras on line.

Arnaut, A. (1994). Historia de una profesión. Maestros de educación primaria en México (1887-1994), México, Centro de Investigación y Docencia Económicas. 
Ezpeleta, J. y Weiss E. (1994). et al.: Evaluación cualitativa del Programa para abatir el rezago educativo, Informe Final. México, Departamento de Investigaciones Educativas del CINVESTAV-IPN.

Ferreiro, E. y Rodríguez, B. (1994). Las condiciones de alfabetización en el medio rural, MÉxico, CINVESTAV- W. K. Kellog Foundation.

Fuenlabrada, I. y Weiss E. (Coordinadores); Candela A.; Ez.peleta, J.: Kalman, J.; Mercado, R. (1997). Las prácticas escolares y docentes en las escuelas unidocente de la educación primaria. Informe Final, México. Departamento de Investigaciones Educativas del CINVESTAV-IPN.

Gobierno de Jamaica. El Programa Externo de la Escuela hacia la Comunidad (SCOPE) Documento publicado en Web el 18 de Agosto 2002. Sitio del Ministerio de Educación y Cultura de Jamaica.

Pontificia Universidad Católica de Chile. Proyectos Operativos sobre Reforma Educativa Chilena Documento en WEB de la Universidad.

Suárez. A. y Rockwell, E. (1978). Evaluación del sistema de cursos comunitarios. México, CONAFE-COPIDER. 